\title{
Overview of the results on divertor heat loads in RMP controlled H-mode plasmas on DIII-D*
}

M.W. Jakubowski ${ }^{1,2}$, T.E. Evans ${ }^{3}$, M.E. Fenstermacher ${ }^{4}$, M. Groth ${ }^{4}$, C.J. Lasnier ${ }^{4}$, A.W. Leonard $^{3}$, O. Schmitz ${ }^{2}$, J.G. Watkins ${ }^{6}$, T. Eich $^{1}$, W. Fundamenski ${ }^{7}$, R.A. Moyer ${ }^{5}$, R.C. Wolf ${ }^{1}$, L.B. Baylor ${ }^{3}$, J.A. Boedo ${ }^{5}$, K.H. Burrell ${ }^{3}$, H. Frerichs ${ }^{2}$, J.S. deGrassie ${ }^{3}$, P. Gohil ${ }^{3}$, I. Joseph ${ }^{5}$, S. Mordijck ${ }^{3}$, M. Lehnen ${ }^{2}$, C.C. Petty ${ }^{3}$, R.I. Pinsker ${ }^{3}$, D. Reiter ${ }^{2}$, T.L. Rhodes ${ }^{5}$, U. Samm ${ }^{2}$, M.J. Schaffer ${ }^{3}$, P.B. Snyder ${ }^{3}$, H. Stoschus ${ }^{2}$, T. Osborne ${ }^{3}$, B. Unterberg ${ }^{2}$, E. Unterberg ${ }^{3}$, W.P. West ${ }^{3}$

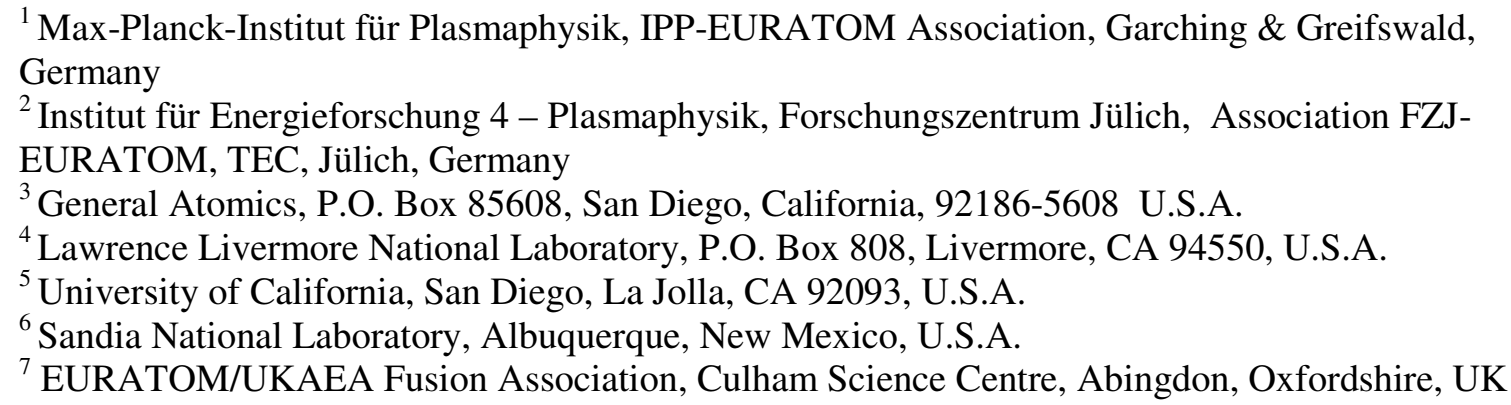

Abstract. In this paper the manipulation of power deposition on divertor targets at DIII-D by application of resonant magnetic perturbations (RMPs) for suppression of large Type-I edge localized modes (ELMs) is analysed. We discuss the modification of the ELM characteristics by the RMP applied. It is shown, that the width of the deposition pattern in ELMy H-mode depends linearly on the ELM deposited energy, whereas in the RMP phase of the discharge those patterns are controlled by the externally induced magnetic perturbation. It was also found that the manipulation of heat transport due to application of small, edge resonant magnetic perturbations (RMP) depends on the plasma pedestal electron collisionality $\nu_{e}^{*}$. We compare in this analysis RMP and no RMP phases with and without complete ELM suppression. At high $\nu_{e}^{*}>0.5$, the heat flux during the ELM suppressed phase is of the same order as the inter-ELM and the no-RMP phase. However, below this collisionality value, a slight increase of the total power flux to the divertor is observed during the RMP phase. This is most likely caused by a more negative potential at the divertor surface due to hot electrons reaching the divertor surface from the pedestal area along perturbed, open field lines and/or the density pump out effect.

\section{Introdction}

Large type-I Edge Localized Modes (ELMs) [1] are a significant concern in tokamak plasmas as they cause high, transient heat loads on the plasma facing components. They appear as a series of rotating filamentary structures [2] due to pedestal pressure gradients found at the edge of H-mode plasmas [3]. These ELM filaments form characteristic spiral heat load pattern on the divertor surface [4]. They have been successfully eliminated in H-mode plasmas at the DIII-D tokamak [5] by application of small, edge resonant magnetic perturbations (RMPs) produced by coils external to the plasma but inside the vacuum vessel over wide range of pedestal collisionalities and plasma shapes [6-9]. In DIII-D a series of coil designs (so called C-coils and I-coils) have been implemented to enhance core plasma performance [10]. Currents flowing in the I-coils create a relatively small magnetic perturbation, which consists mainly of components with $n=3$ and is resonant to the q=3 flux surfaces, located at the

\footnotetext{
Work supported by the US Department of Energy under DE-AC04-94AL85000, DE-AC5207NA27344, DE-FC02-04ER54698 and DE-FG02-04ER54758.
} 
plasma boundary for the plasmas investigated. Depending on the current distribution within the coils one can realize magnetic perturbation with different up/down symmetry. The parity of the I-coils is named 'even' when the upper and lower coils have the same polarity at each toroidal angle $\varphi$ and 'odd' when these polarities are opposite. From the vacuum modelling it follows that 'even' parity creates magnetic perturbation with higher pitch resonance, i.e. higher stochastization of the magnetic field lines.

The interaction of the RMP with magnetic equilibrium imposes a three-dimensional topology of perturbed magnetic field lines in the plasma edge [11]. Topologically, this volume consists of two layers:

1. An inner stochastic boundary, where the radial particle transport is potentially enhanced due to high diffusivity of magnetic field lines $[12,13]$ and non-ambipolar particle transport for instance [14,15], is located near the pedestal area. It has been shown in [16] that the suppression of Type-I ELMs correlates with a minimum width of the edge region having magnetic islands with Chirikov parameter $>1.0$, based on vacuum calculations of RMP mode components excluding the plasma response or rotational shielding.

2. In a diverted tokamak, poloidal magnetic flux $(\psi)$ escaping the outer edge of this stochastic region is organized by complex topological structures known in nonlinear dynamical systems theory as homoclinic tangles [17]. Such a tangle is generated by a splitting of the separatrix into a set of invariant manifolds when perturbed by small nonaxisymmetric magnetic fields. At the separatrix a set of invariant manifolds is created, which forms a specific spiral pattern on the divertor surface. Lobes of the manifolds form an envelope for the open field lines leaving the stochastic area and intersecting the divertor target plates [18]. Those field lines are mixture of long connection length stochastic field lines and short connection length laminar field lines. On the target surface lobes form striated substructures of the strike line.

As the parallel transport exceeds by orders of magnitudes the perpendicular one, it is expected that during the RMP phase of the discharge the heat and particle flux on the divertor target plates should reflect this generic structure of the striated strike lines. This has been observed in different experiments including limiter [19-21] and divertor plasmas [17,22]. The aim of this paper is to discuss effects of the stochastic boundary at DIII-D on the heat loads to the divertor surface. It consists of two parts:

- In section 3, which follows discussion of the experimental set-up, we discuss the properties of ELM heat load deposition patterns and their modification by the $n=3$ external perturbation. The investigations have been performed in plasmas with ITER Similar Shape and electron pedestal collisionalities. It has been found, that the wetted area of ELMs during non RMP H-mode discharge depends on ELM size in terms of the power deposited to the inner divertor leg. This is consistent with recently proposed mechanism for ELM behaviour given in [23], that temperature losses due to an initial peeling-ballooning instability, conducted along opened magnetic field lines due to a small pre-existing perturbation leads to a temperature difference at the end point of the field lines at the inner and outer divertor targets. The resulting thermo-electric currents are supposed to be capable to amplify explosively the existing magnetic field perturbation. Moreover, we do see that remaining ELMs in the initial RMP phase, have very similar target power load deposition patterns. This suggests that ELMs are controlled by the external field and their power deposition patterns resemble the structure of magnetic footprints on the target calculated with the TRIP3D code [24].

- This initial relatively short period, of RMP interaction with ELMs is followed by the phase when ELMs are completely eliminated. It is quite important for the ITER safety to understand how the power flux changes in this case. In section 4 we review the 
results of heat flux behaviour with the $n=3 \mathrm{RMP}$ and compare them to non-RMP Hmode results for a wide range of pedestal electron collisionalities $\nu_{\mathrm{e}}^{*}=q_{95} R \varepsilon^{-3 / 2} \lambda_{\mathrm{ee}}^{-1}$ at high and low triangularity (including ITER-similar shapes). Here $R$ is the major radius of the torus, $\varepsilon \equiv a / R$ inverse aspect ratio and $\lambda_{\mathrm{ee}}$ is the mean free path for electron collisions. This analysis includes the power balance, inner/outer leg asymmetries and structure of the strike lines. Switching on I-coils in plasmas with higher $\nu_{\mathrm{e}}^{*}$ does not affect power deposited to the divertor independent on the I-coils parity. At ITER-like collisionalities we observe small increase of the heat flux to the target plates. Also, as expected, double null plasmas show higher asymmetries than single null discharges. Ratio of inner to outer power loads shows dependence on electron pedestal collisionality.

\section{Experimental set-up}

We examine in this paper both, quasi double-null plasma discharges at high $\nu_{e}^{*}$ with moderate to high triangularity as well as lower single null plasmas with low density, low $\nu_{e}^{*}$ (both low and high triangularity). The plasma shapes discussed within this work are presented in Figure 1. Main parameters for all the discharges discussed within this work are presented in Table 1. Between the 2005 and 2006 operating period the DIII-D lower cryopump baffle was extended, as shown in Figure 1c, to allow for optimized pumping in plasmas with higher triangularities - similar to those planned for ITER. All those discharges had slight variation in $q_{95}$ in order to stay in the resonant window for magnetic perturbations, in which Type-I ELMs are completely suppressed [16].

\begin{tabular}{|c|c|c|c|c|c|c|}
\hline Discharge & $\begin{array}{c}\text { Plasma } \\
\text { current } \\
I_{\mathrm{p}}[\mathrm{MA}] \\
\end{array}$ & $\begin{array}{c}\text { Toroidal } \\
\text { field on axis } \\
B_{\varphi}[\mathrm{T}] \\
\end{array}$ & $\begin{array}{c}\text { Upper/lower } \\
\text { triangularity } \\
\delta_{\mathrm{U}} / \delta_{\mathrm{L}} \\
\end{array}$ & $\begin{array}{c}\text { Electron pedestal } \\
\text { collisionality } \\
\nu_{\mathrm{e}}^{*} \\
\end{array}$ & $\begin{array}{c}\text { I-coil } \\
\text { current } \\
I_{\text {coil }}[\mathrm{kA}] \\
\end{array}$ & $\begin{array}{l}\text { I-coil } \\
\text { parity }\end{array}$ \\
\hline 115456 & 1.14 & -1.62 & $0.4 / 0.8$ & 2 & 4.5 & odd \\
\hline $\begin{array}{l}119690 \\
119692 \\
119695 \\
\end{array}$ & 1.05 & -1.62 & $0.4 / 0.6$ & 3 & 4.2 & odd \\
\hline 119902 & 1.18 & -1.63 & $0.4 / 0.7$ & 1.3 & 4.5 & odd \\
\hline 122461 & 1.11 & -1.62 & $0.4 / 0.7$ & 0.5 & 6.4 & even \\
\hline 122462 & 1.17 & -1.77 & $0.4 / 0.6$ & 0.5 & 6.4 & even \\
\hline 122465 & 1.42 & -1.93 & $0.2 / 0.4$ & 0.5 & 6.4 & even \\
\hline 122342 & 1.41 & -1.92 & $0.2 / 0.4$ & 0.4 & 3.0 & even \\
\hline $\begin{array}{l}123301 \\
123306\end{array}$ & 1.53 & 2.01 & $0.2 / 0.4$ & 0.3 & $2-3 \mathrm{kA}$ & even \\
\hline 126006 & 1.55 & -1.94 & $0.3 / 0.7$ & 0.2 & 4 & even \\
\hline $\begin{array}{l}129194 \\
129197\end{array}$ & 1.52 & -1.85 & $0.3 / 0.7$ & 0.2 & 4.5 & even \\
\hline
\end{tabular}

Table 1. Overview of the main parameters of the discharges discussed within the text.

The preferred diagnostic for divertor target heat load measurements is infra-red thermography. In this work the heat flux analysis has been performed with two infra-red cameras mounted at two different toroidal locations: 1) a fast-framing infra-red Santa Barbara Focal Plane (SBF-125) camera [25] at toroidal angle of $\left(\varphi=165^{\circ}\right)$ with a time resolution of $13 \mathrm{kHz}$ and spatial resolution of 5-7 millimetres per pixel on the target surface, and 2) the FLIR Systems Inc. infra-red camera located at toroidal angle $\left(\varphi=60^{\circ}\right)$ with $50 \mathrm{~Hz}$ time resolution and similar spatial resolution. Both cameras have a viewing geometry observing the lower divertor area; however in most cases they did not run simultaneously. The setup for 
the infrared camera SBF-125 is presented in Figure 2a. Both cameras observe the surface of the lower divertor using similar optics. As presented in Figure $2 \mathrm{~b}$ a toroidal region of $\Delta \varphi \approx 25^{\circ}$ centered at $\varphi=135^{\circ}$ is observed by the SBF system. At present in most plasma configurations only the inner strike line can be observed due to the shape of the pumping duct, which covers the outer strike line from the camera view. However for most of the discharges discussed in section 4 the infra-red data was taken with both strike lines visible due to a different geometry of the lower divertor shelf (see Figure 1a-b).

The heat fluxes on the target surfaces are calculated for the SBF-125 system by applying a standard numerical solution of the two-dimensional heat diffusion equations to the evolution of the surface temperature on the investigated area with the THEODOR code [26], while for the FLIR system a semi-infinite approximation for the heat diffusion in a solid bulk material is used. The THEODOR code, which is used to study heat loads due to Type-I ELMs has the ability to evaluate the influence of the surface layers on the evaluated heat flux density. The code starts from the temporal evolution of the surface temperature distribution along the poloidal target coordinate obtained with help of the LEOPOLD code [4] and computes the heat flux distribution using a 2D slab geometry approximation for the target tiles, introducing the real poloidal target width and an averaged target thickness. Front surface layers are taken into account with the heat transmission coefficient $\alpha$, which is chosen in such a way that negative heat fluxes in the inter-ELM period are avoided. In this work $\alpha=40 \mathrm{kWm}^{-2} \mathrm{~K}^{-1}$ has been set for the investigated discharges. The coefficient assumes the same surface properties across the strike line, which is not necessarily correct; therefore some caution must be taken with absolute numbers of the heat flux density. A detailed discussion of the method on examples of ASDEX-Upgrade and JET data is given in [26,27]. In the section 4 we use low time resolution camera data to discuss global changes of the power flux caused by the RMP, therefore a semi-infinite approximation, which does not take into account surface layers is sufficient. Additionally, the data is checked against global power balance; we find fair agreement between heat fluxes obtained from both methods.

\section{Influence of RMP on ELM deposition patterns}

It has been reported from ASDEX-Upgrade, that Type-I ELMs create helical footprint patterns of heat flux on the divertor surface [4]. Several strike lines were detected outside the original strike point of the outer leg albeit at very low amplitude. This is often referred as a striation of the strike line, although it can be either due to strike line splitting or as stated in [4] different filaments hitting a surface of the divertor target plates. Filaments form helically aligned structures, which are clearly related to the topology of the magnetic field. In this work we have found very similar structures on the inner and outer target plates. However, as most of the area of the outer strike point is hidden from the infrared camera by the vessel structures, we will concentrate on the substructures detected during ELM events on the inner target plates of an $\mathrm{H}$-mode discharge. The investigated discharges have been performed at low pedestal collisionality $\left(\nu_{\mathrm{e}}^{*} \approx 0.3\right)$ and ITER-like plasma shapes $\left(\delta_{U}=0.32, \delta_{L}=0.70\right)$. An example of the scenario is given in

Figure 3. At $t \approx 1200 \mathrm{~ms}$ there is a transition to ELMy H-mode associated by a mixture of large Type-I ELMs with frequency of about $50 \mathrm{~Hz}$ and small ones (probably Type-II ELMs [6]). The maximum power load to the inner target during an ELM can reach up to $15 \mathrm{MW} / \mathrm{m}^{2}$ for the largest Type-I ELMs and about 2-3 MW/m² for the smaller ELMs. Switching on the Icoil current of $4.5 \mathrm{kA}$ at first makes ELMs more frequent $(\sim 200 \mathrm{~Hz})$ and changes their amplitudes. Peak heat flux due to Type-I ELMs decreases roughly by factor of 3 and due to Type-II increases by factor of 2 . Shortly before $\mathrm{t}=2400 \mathrm{~ms}$ all instabilities disappear completely. Small ELM-like bursts at $\mathrm{t} \approx 2500 \mathrm{~ms}$ are caused by pellets injected into the 
plasma. They do not appear in every discharge and have amplitudes slightly smaller than ELMs in the initial RMP phase, i.e. much smaller than the ELMs without the RMP.

\subsection{Basic properties of ELMs}

Before discussing ELM deposition patterns during the initial RMP phase, we present observations from the non-RMP H-mode phase of the discharge inspected. An example of an infrared image taken by the SBFP camera of the substructures during an ELM without the RMP is shown in Figure 4. Three additional strike lines are visible in the remote area of the inner strike line (upper, right part of the image). Usually, there are two to five nonaxisymmetric strike lines observed at the inner strike point position during an ELM event. Their width is typically in the range of $2-4 \mathrm{~cm}$ and the separation between them is of the same order. The grid projected onto the image shows the vessel model applied in the LEOPOLD code [4] to unfold the temperature data.

For the purpose of this work we have performed calculations in the area indicated by two yellow dashed lines. Temperature data have been obtained with very good time resolution $(f=13 \mathrm{kHz})$, which allowed to study the evolution of the structures during an ELM event. Two examples are shown in Figure 5. In the example on the left hand side - at the ELM onset ( $t=$ $1961 \mathrm{~ms}$, \#129197) two strike lines appear (at $s-s_{0} \approx 30 \mathrm{~mm}$ and $s-s_{0} \approx 90 \mathrm{~mm}$ ) with the latter one depositing most of the heat to the target. Roughly $80 \mu$ s later, a third substructure (at $s-s_{0} \approx 50 \mathrm{~mm}$ ) appears. All the structures decay within $2 \mathrm{~ms}$ to the pre-ELM values. It has been reported, e.g. in [2], that Type-I ELM filaments rotate poloidaly/toroidaly. For some of the ELM events there is a signature of the lobes separation, which could be interpreted as a rotation of the structures. An example of such behavior is shown in Figure 5b. The time scales of the events recorded by the camera are very similar to the previous case, the difference is however in the internal evolution of the substructures. Here we observe an increasing separation of the main strike line and the second lobe from $\Delta s \approx 50 \mathrm{~mm}$ to $\Delta s \approx 110 \mathrm{~mm}$ within the time frame of $100 \mu \mathrm{s}<\Delta t<500 \mu \mathrm{s}$, at the end of which the peak heat load reaches its maximum of $7 \mathrm{MW} / \mathrm{m}^{2}$. As we expect that the ELM filaments produce spiraling patterns on the target [28], an increase of the separation of the lobes is consistent with an assumption of toroidal movement of the striated patterns. After $\Delta t=500 \mu$ s again we do not see any evolution of the pattern on the target, but only a decay of the heat load to the pre-ELM state. As we perform a measurement at one toroidal location we are not able to derive toroidal velocity of the rotation.

The energy deposited to the inner target per ELM for all investigated ELMs is presented in Figure 6 as a function of the time of the ELM event. Each point represents the energy deposited to the inner target during one event. It is calculated with the following formula

$$
E_{\mathrm{dep}}=2 \pi \int_{s_{0}}^{s_{\mathrm{n}}} \int_{t_{0}}^{t_{\mathrm{n}}} q(s, t) R(s) d s d t-E_{\mathrm{base}},
$$

where $q(s, t)$ is the heat flux density, $s_{0}$ and $s_{\mathrm{n}}$ define spatial limits for the integration, $t_{0}$ and $t_{\mathrm{n}}$ - starting and ending time point of single ELM event and $R(s)$ is a major radius. $E_{\text {base }}$ is the inter-ELM power to the target, which would be deposited to the inner target if there would be no ELM in this time range; it is interpolated from $\mathrm{E}\left(t_{0}\right)$ and $E\left(t_{n}\right)$. Both time limits has been chosen based on the results of [27], i.e. $t_{0}$ just before the heat pulse reaches target plates and $t_{\mathrm{n}}$ - when averaged heat flux reaches its minimum in the tail of an ELM. Results reveal two groups of data points, which are well separated from each other and marked with blue and red circles. These are identified as Type-I and, probably, Type-II ELMs respectively. The latter ones, as expected, deposit much smaller energy to the inner target (below $10 \mathrm{~kJ}$ ). Before the RMP phase $(\mathrm{t}<2200 \mathrm{~ms})$ one observes an increasing amount of energy deposited to the target per ELM for $1200 \mathrm{~ms}<\mathrm{t}<2100 \mathrm{~ms}$. This is caused by increasing heating power from the neutral beam injection, which reaches a maximum of $9 \mathrm{MW}$ at $\mathrm{t} \approx 2100 \mathrm{~ms}$. At this time the 
energy deposited per ELM reaches $40 \mathrm{~kJ}$ for Type-I and a few $\mathrm{kJ}$ for Type-II ELMs. Application of RMP at $t=2200 \mathrm{~ms}$ reduces the amount of energy deposited per event for Type-I ELMs to the value in the range of $20-30 \mathrm{~kJ}$. Surprisingly, Type-II ELMs deposit more energy with the magnetic perturbation $(5-10 \mathrm{~kJ})$ than without the RMP. Bursts caused by the injection of $D_{2}$ pellets recreate both types of ELMs for a short period of time at $t \approx$ $2500 \mathrm{~ms}$.

\subsection{Variation of ELM deposition footprints with ELM size}

One of the recent ideas [23], which still needs experimental confirmation, about the evolution of the Type-I ELMs assumes that the transient event is initiated when a peelingballooning mode gets destabilized as the pedestal pressure gradient exceeds the linear marginal stability limit of the mode [29]. This produces an initial pulse of heat and particles that propagates radially outward into a small pre-existing homoclinic separatrix tangle. Due to different parallel connection length from the outboard midplane to the inner and outer targets the increase of the electron temperature at the outer strike point is higher than at the inner one, which leads to the onset of thermoelectric currents within the filaments [30,31]. Theoretically there are only two possible sources available to drive these currents: the thermoelectric effect and the Pfirsch-Schlütter (parallel pressure gradient) effect [31]. Both are probably present during the ELM cycle but theoretical estimates suggest that the thermoelectric current should be dominant based on the large heat flux released by the ELM [32]. On the other hand there is a recent measurement from ASDEX-Upgrade, which suggests that the sign of the current is not compatible with the thermoelectric nature of those currents [30]. Also it is shown that, the difference between the ELM energy load on the inner and outer target is well correlated with the measured charge flowing through the targets due to the ELM.

Independent on the nature of the currents flowing within the filaments - as they have helical form - their currents should create a magnetic perturbation, which most likely includes Fourier components that are resonant to the edge magnetic equilibrium. It has been shown that divertor heat flux and particle recycling patterns are consistent with magnetic footprints produced by separatrix splitting associated with homoclinic tangles under some conditions $[22,24]$. Since homoclinic tangles result naturally from a variety of stationary and/or time dependent non-axisymmetric magnetic perturbations that are found in any realistic tokamak due to many sources of field errors [24,31,32], it is not unreasonable to expect these structures to be the norm rather than the exception, also during the on-set of an ELM. These components should create at least a temporal perturbation of the separatrix, which is consistent with the observed splitting of the separatrix during ELMs. Intuitively, one would expect that larger ELMs should carry larger currents, which would eventually lead to the greater splitting of the separatrix as compared to small ELMs. This is actually consistent with the experimental findings as presented below. Although we could not perform accurate comparison of the heat loads in the discharges discussed in this work, there is an evidence for the inner/outer asymmetries of the heat loads [33].

The width of an ELM deposition pattern is defined here as

$$
w_{f}=\frac{\left.\int q(s)\right|_{t=t_{\max }} d s}{\max \left(\left.q(s)\right|_{t=t_{\max }}\right)}[\mathrm{m}],
$$

where $q\left[\mathrm{MW} / \mathrm{m}^{2}\right]$ is the heat flux density profile taken at the time when the heat load to the divertor reaches its maximum $\left(t=t_{\max }\right), s[\mathrm{~m}]-$ is the coordinate along the divertor contour, and $\max (q(s))$ denotes the maximum of the heat flux density profile. ELM wetted area, i.e. area, where the heat flux is deposited, is proportional to $2 \pi R w_{f}$ (with $R$ being major radius of the strike line). The dependence of $w_{f}$ on ELM size, expressed as a function of deposited 
energy $E_{\text {dep }}$, is shown in Figure 7. There is a clear increase of the width versus the amount of energy deposited per ELM from about $4 \mathrm{~cm}$ for Type-II ELMs $(<10 \mathrm{~kJ})$ to about $8 \mathrm{~cm}$ for the largest Type-I ELMs. Below $30 \mathrm{~kJ}$ the increase seems to have a linear characteristic, but it saturates for $E_{\mathrm{dep}}>30 \mathrm{~kJ}$. It is most likely caused by the deposition on the center post of the tokamak. The values of $w_{\mathrm{f}}$ are slightly smaller than the profile widths at the half-maximum. Profiles of deposited power for different ELMs are presented in Figure 8a. They are ordered according to the energy deposited to the target (the abscissa) and normalized to the maximal value for each of the profiles. Here again, one recognizes increasing width of the deposition pattern with the ELM size. Also a number of detected peaks in the profile structure increases (as shown in Figure 9a). Small instabilities show 2-3 peaks in their profiles at the maximum of the heat deposition. Larger instabilities show more random structuring, with typically 3 or 4 substructures (15 out of 19 ELMs). There are few ELMs, which show either five substructures (1) or less then three (3). This variability is caused most likely by a different toroidal phase of the footprint at the moment of detection and/or different shape of the perturbation spectrum. One should note, that large changeability of the spatial distribution of those substructures indicates that they should not be the artifacts of the surface layers. In case of the layers producing bright stripes in the infrared image, the position of those stripes would be the same for all ELMs. As it can be seen in Figure 8a, we do not observe stripes, which appear for all of the investigated ELMs, i.e. we do not expect the observed striation to be caused by the heterogeneous structure of the surface layers.

A very interesting change in ELM behavior happens, when the $n=3$ RMP plasma operation is considered (red dots in Figure 7). For both types of ELMs data points become less spread in term of ELM size and wetted area for the Type-II ELMs. Wetted areas $\left(w_{\mathrm{f}} \approx 3-4 \mathrm{~cm}\right.$ and 5-8 $\mathrm{cm}$ respectively) become narrower (if we neglect one ELM with Edep $\approx 21 \mathrm{~kJ}$, wf $\approx 9 \mathrm{~cm}$ ), when compared to the same energies in the non-RMP case. As presented in Figure $8 \mathrm{~b}$ and Figure $9 \mathrm{~b}$ their structure became almost the same - most of the instabilities with energy deposited below $15 \mathrm{~kJ}$ have either any additional substructure or one additional but rather faint. On the other hand 64\% (7 out of 11) of the ELMs with energy deposited above $20 \mathrm{~kJ}$ show three lobes with the distance along them not varying with the energy. This probably means that structure of the separatrix is dominated by the magnetic perturbation coming from the I-coils, i.e. that the radial magnetic component of the magnetic field induced by $4 \mathrm{kA} \mathrm{I-}$ coil current is much stronger, than $b_{\mathrm{r}}$ induced by currents flowing within the filaments. Indeed, measurements performed on DIII-D have shown that filaments can carry currents of order of hundreds of Amperes [34]. The heat pulse due to ELMs travelling across the separatrix is diverted along the open field lines of the stochastic boundary to the target plates and deposited through the lobes of the separatrix. Figure 10 shows a comparison of the measured heat flux density profile with predictions of the structure of target patterns from TRIP3D. When we compare the deposition profile of Type-I ELM with the topology of magnetic footprints we find fair agreement between both structures. Additionally those structures do not show the same dynamics as ELMs in the non-RMP phase of the discharge. Figure 11 depicts an example of the power deposition pattern during a Type-I ELM in the same manner as in Figure 5. The heat pulse reaches first the main strike line and after $100 \mu \mathrm{s}$ simultaneously two lobes at $\Delta s \approx 60 \mathrm{~mm}$ and $\Delta s \approx 115 \mathrm{~mm}$. All three hot spots reach their maximum at $\Delta t \approx 200 \mu \mathrm{s}$. In contrary to non-RMP results from Figure 5 , here we do not observe changes of the hot spots structure. Energy deposition through the external substructures decays much faster as for the main strike line. They show no signature of any filament rotation, which suggests locking of ELMs to the external perturbation. For all investigated instabilities the maximum of the power deposition falls into the main strike line and decays with increasing distance from the separatrix. This picture qualitatively resembles 
results of numerical modeling of heat transport in weakly stochastic fields performed with EMC3-EIRENE [11] and E3D [35] codes. Both codes predict "filling" of the outer separatrix lobes with energy coming from the pedestal by stochastic field lines in the case of static magnetic perturbations. This is consistent with the hypothesis presented above about the stochastic nature of the heat transport during an ELM. Most likely there is a positive coupling of the resonant magnetic components coming from the filament currents and the I-coils, that enhances the heat transport through the pedestal. One should note that the ratio of power deposited through the outer lobe to the main strike line is much higher than in the case of the ELM-free RMP phase of the discharge (see Figure 15).

\section{Heat flux to the divertor at different collisionalities}

The heat flux data presented in this section is recorded with time resolution of $50 \mathrm{~Hz}$, which makes it impossible to resolve details of heat loads for each individual ELM. The data for ELMs recorded with fast framing camera was discussed in previous section. Here we concentrate on global changes in the power deposited to the divertor when ELMs are suppressed by means of a resonant magnetic perturbation. High and low $\nu_{\mathrm{e}}^{*}$ discharges show different behaviour of the heat flux to the divertor during ELM suppression. Two examples are presented in Figure $12 \mathrm{a}$, where time traces for \#119692 $\left(\nu_{\mathrm{e}}^{*} \approx 3-4, \quad q_{95}=3.7\right)$ and \#126006 $\left(\nu_{\mathrm{e}}^{*} \approx 0.3, \quad q_{95}=3.5\right)$ are presented. The discharges are performed with upper and lower triangularity of $\left(\delta_{\mathrm{up}}=0.4, \delta_{\mathrm{low}}=0.6\right)$ and $\left(\delta_{\mathrm{up}}=0.3, \delta_{\mathrm{low}}=0.7\right)$ respectively. In both cases the I-coils have been run with almost the same current of $4 \mathrm{kA}$. The currents have been run with either even (\#126006) or odd (\#119692) parity.

In the high $\nu_{\mathrm{e}}^{*}$ case (black curves in Figure 12a) ELMs are suppressed immediately after the Icoils are switched on $(t=3000 \mathrm{~ms})$. There is no significant effect on the heat flux to the target plates of the lower divertor during the whole RPM phase. As the diamagnetic energy is not affected by stochastization of the plasma boundary, there is also no temporary increase of the power flux due to a heat pulse crossing the separatrix. As reported in [36], at high collisionalities, the floating potential $\left(V_{\mathrm{f}}\right)$ measured by the divertor Langmuir probes near the outer strike point is approximately zero during application of the RMP, suggesting a drop of the temperature in the scrape-off layer and weaker acceleration of ions towards the target by the sheath due to RMP application. For discharge \#119692, the floating potential increases from slightly above zero to a few volts as shown in Figure $12 b$, black curve, i.e. there is no significant change of sheath properties.

Contrary results are obtained for low collisionality discharges (e.g. \#126006 in Figure 12a) ELM suppression by the RMP is associated with a density pump-out and a small drop of the diamagnetic energy. As the confinement drops there is an additional portion of energy leaving the plasma, which is immediately seen as a 50\% increase of the total heat flux to the divertor. After the energy confinement reaches its new equilibrium, the energy deposited to the divertor remains on a higher level as compared to the non-RMP phase (70\% of the total power on average in \#126006 as compared to $60 \%$ on average in pre-RMP phase). This increase of the heat flux is observed for all investigated discharges with $\nu_{\mathrm{e}}^{*}<0.5$ at DIII-D independent on the plasma triangularity. It is accompanied by the fact that the floating potential at the position of the strike point becomes strongly negative as illustrated in Figure 12b, which suggests interaction of hot electrons with the surface of the target plates. Most likely these electrons come from the pedestal area along the magnetic field lines perturbed by the RMP and at low $\nu_{e}^{*}$ they are almost collisionless. Conversely, at $\nu_{e}^{*}=3$ or 4 they are not able to reach to the sheath area from the pedestal, i.e. the hot electrons are more likely to dissipate energy before reaching the surface of the targets. A more negative floating potential enhances the transfer of 
energy to ions and thus increases the heat flux to the surface. For all the investigated cases with $v_{\mathrm{e}}^{*} \gtrsim 0.5$ independent on the parity of the I-coils, there is no permanent increase of the heat flux. Figure 13 illustrates the changes in the power reaching the divertor $\left(P_{\text {target }}\right)$ and the radiated power $\left(P_{\text {rad }}\right)$ normalized to the total heating power $\left(P_{\text {total }}\right)$ when the I-coils are energized. The power to the target is obtained using the equation:

$$
P_{\text {target }}^{\text {calc }}=P_{\text {total }}-P_{\text {rad }}-\frac{\mathrm{d} W_{\text {dia }}}{\mathrm{d} t},
$$

where $W_{\text {dia }}$ is the diamagnetic stored energy. For all the cases, where inner and outer legs could be observed by the infrared camera (e.g. \#119692 - Figure 13a), there is a very good agreement between $P_{\text {target }}$ and power to the divertor measured by the camera. At low collisionality, before the RMP phase, about $70 \%$ of the total energy is deposited to the target. This amount is increased by about $15 \%$ (to $80 \%$ of total heating power) after the I-coils are energized; at the same time radiated power drops from $25 \%$ to $20 \%$ of $P_{\text {tot }}$. The situation changes for higher $\nu_{e}^{*}$ - there both $P_{\text {target }}$ and $P_{\text {rad }}$ exhaust a similar fraction of energy, which is not affected by the RMP. The exact values of the radiated power are given in Table 2.

\begin{tabular}{|c|c|c|c|c|}
\hline \multirow[b]{2}{*}{ Volume } & \multicolumn{2}{|c|}{$\# 119692\left(\nu_{\mathrm{e}}^{*} \approx 3\right)$} & \multicolumn{2}{|c|}{$\# 126006\left(\nu_{\mathrm{e}}^{*} \approx 0.2\right)$} \\
\hline & $\begin{array}{c}t=2600 \mathrm{~ms} \\
(\mathrm{no} R M P)\end{array}$ & $\begin{array}{c}\mathrm{t}=3750 \mathrm{~ms} \\
(\mathrm{RMP})\end{array}$ & $\begin{array}{c}\mathrm{t}=1775 \mathrm{~ms} \\
(\mathrm{no} \mathrm{RMP})\end{array}$ & $\begin{array}{c}t=2500 \\
(\mathbf{R M P})\end{array}$ \\
\hline Core & $0.63 \mathrm{MW}$ & $0.84 \mathrm{MW}$ & $0.54 \mathrm{MW}$ & $0.69 \mathrm{MW}$ \\
\hline SOL & $0.33 \mathrm{MW}$ & $0.39 \mathrm{MW}$ & $0.42 \mathrm{MW}$ & $0.23 \mathrm{MW}$ \\
\hline Lower divertor (inner leg) & $0.42 \mathrm{MW}$ & $0.37 \mathrm{MW}$ & $0.50 \mathrm{MW}$ & $0.41 \mathrm{MW}$ \\
\hline Lower divertor (outer leg) & $0.53 \mathrm{MW}$ & $0.53 \mathrm{MW}$ & $0.30 \mathrm{MW}$ & $0.22 \mathrm{MW}$ \\
\hline Upper divertor (inner leg) & $0.04 \mathrm{MW}$ & $0.04 \mathrm{MW}$ & - & - \\
\hline Upper divertor (outer leg) & $0.14 \mathrm{MW}$ & $0.09 \mathrm{MW}$ & - & - \\
\hline Total & $2.09 \mathrm{MW}$ & $2.26 \mathrm{MW}$ & $1.75 \mathrm{MW}$ & $1.55 \mathrm{MW}$ \\
\hline
\end{tabular}

Table 2. Overview of the changes in the radiated power due to RMP for the discharges \#119692 and \#126006. The values are obtained from bolometric data and have about $10 \%$ error bar.

In both cases I-coil currents cause an enhancement of radiation by roughly $30 \%$ in the plasma core. The difference is clearly visible at the plasma boundary, where in high collisionality case radiated power is slightly increased in the scrape-off layer and almost not affected in the divertor volume. At low collisionality we observe about $50 \%(0.2 \mathrm{MW})$ drop of $P_{\text {rad }}$ in the scrape-off layer and about $25 \%(0.2 \mathrm{MW})$ in the divertor area. All these findings indicate that the sheath properties are changed by application of RMP leading to the enhancement of the energy transfer to the target plates. A lower floating potential leads to a higher rate of energy deposited by the ions in the sheath and decreases the radiated power in the scrape-off layer. At higher collisionalities, high energy electrons dissipate energy by collisions and do not affect the sheath potential so effectively. Thus the total amount of power deposited to the divertor surface is not affected by the RMPs. Probably, the pre-RMP ratio of $P_{\text {rad }}$ and $P_{\text {target }}$ in low collisionality discharges can be maintained during the RMP phase by seeding of a small fraction of impurities, which would enhance radiation in the divertor area.

\subsection{Properties of the inner and outer strike lines in ELM suppressed phase of the discharge.}

As mentioned already in Section 1, application of any non-axisymmetric perturbation removes degeneration of the separatrix manifolds, what is often referred to in the literature as 
a splitting of the strike line. It has been reported previously that the particle flux, contrary to the heat flux, almost always creates measurable signature of the perturbed strike line striation (see e.g. [11]). For the discharges investigated in this work, we observe that the structure of the heat flux profiles on the divertor surface changes with the pedestal collisionality, which has been observed as well in limiter plasmas at TEXTOR [37]. Figure 14a shows profiles of normalized heat flux density measured at the location of the outer strike point during the RMP phase at four different pedestal collisionalities. ELMs are suppressed in all four discharges. It is apparent that at high $\nu_{\mathrm{e}}^{*}$ an additional lobe of the strike zone appears, when the $n=3$ perturbation is applied. It has been found that there is only a clear splitting of the separatrix visible in the heat flux at high collisionalities $\left(\nu_{\mathrm{e}}^{*}>2\right)$. However, as discussed in [38] the separation of the lobes is 2-3 times larger than anticipated by the TRIP3D calculations [10] depicted for the high $\nu_{\mathrm{e}}^{*}$ case (\#119692) in Figure 14b and for the low $\nu_{\mathrm{e}}^{*}$ case in Figure 14c. In the latter case, only very small heat fluxes [22] and in the actual example no heat flux at all is seen to be channeled along the perturbed separatrix lobes although they are separated by a rather huge distance of $1.5 \mathrm{~cm}$ each (see Figure 14c).

These results suggest that the plasma response to the applied $n=3$ edge resonant magnetic perturbation may amplify the effects of the external perturbation in terms of perturbation of the separatrix manifolds. This has two effects on the power deposition pattern to the divertor:

a. Amplification of the separatrix deformation, i.e. wider splitting of the strike line than predicted from the vacuum magnetic field modeling.

b. Possible enhanced coupling of the outer lobes to the pedestal by deeper penetration of magnetic field lines, which results in higher heat deposited through the outer lobes. One should note, that enhanced heat flux in the outer lobe also happens during discharges affected by locked modes in the plasma core and (as discussed in the next section) during ELMs.

At lower collisionalities (e.g. \#123301) the width of the heat flux profile ( $3 \mathrm{~cm}$ ) corresponds roughly to the structure of the outer strike point (see Figure 14c) calculated by the TRIP3D code, which has about $3 \mathrm{~cm}$ width at $\varphi=60^{\circ}$. As the thermographic system has a spatial resolution of order $1 \mathrm{~cm}$, it is not possible to evaluate the internal structure of the strike point. However, recent measurements with higher spatial resolution show that the outer lobe of the inner strike line during discharges with $\nu_{\mathrm{e}}^{*} \approx 0.2$ can be resolved experimentally, but with a low amount of the heat deposited there. This is presented in Figure 15, which is reproduced from [22]. This scenario utilizes plasma with ITER Similar Shape and ITER-like electron pedestal collisionality, and with I-coil currents of $4 \mathrm{kA}$. An overlapping region on the lower divertor surface is observed simultaneously using a CCD camera with a set of filters suitable to get spectral lines strongly related to the incoming particle flux and the IR camera SBF-125. Direct comparison of particle fluxes with the connection length profile shows three lobes of the striated strike line, with a shape in good agreement to the TRIP3D predicted structure of magnetic footprints, what is consistent with TEXTOR results on the stochastic boundary [37,39]. In the heat flux (Figure 15c) only the innermost of the separatrix lobes forms a large local heat flux maximum. The second lobe, detected well in particle flux, is hidden in the shoulder of the main heat flux peak, and the third lobe carries a power load with a peak value of about $0.4 \mathrm{MW} / \mathrm{m}^{2}$. As discussed in [11] these results confirm thermal connection between plasma pedestal and the target plates with relatively short connection length field lines.

\subsection{Target energy deposition in/out asymmetry}

It is a well known effect in poloidally diverted tokamaks that there is a power load asymmetry between the inner and outer leg of the divertor. For the inter-ELM heat loads this is caused by the $\vec{E} \times \vec{B}$ drifts, ballooning transport on the low field side and geometrical effects [40,41]. 
Results from JET show that the power loads of low triangularity discharges are almost identical to both legs of the divertor at pedestal collisionalities below $0.1\left(P_{\text {inner }} / P_{\text {outer }} \approx 1\right)$, while the ratio of the powers to the inner and outer leg of the divertor decreases to a value of $P_{\text {inner }} / P_{\text {outer }} \approx 0.1$ at a electron pedestal collisionality of 0.5 . In this section we study the asymmetry in the ELM suppressed phase of the discharge. The results are shown in Figure 16. The data have been obtained for plasmas with either high (closed points) or low triangularity (open points) and averaged over the whole RMP phase. The general tendency is that the low triangularity plasmas show lower asymmetries as the discharges with high triangularity. This is not a surprising result as high triangularity plasmas are quasi double null plasmas. This results in some power deposition on the plasma facing components of the upper divertor due to the ballooning which localizes the transport rather on the low field side. Here we do observe that about $30-40 \%$ of the total deposited power goes to the inner target plate at pedestal collisionalities of order of $0.2-0.4$. There is no any clear tendency here to be observed as the data have rather large scatter. The values are of the same order as the results from JET [27] for the inter-ELM phase; however we do not observe such a strong dependence on electron pedestal collisionality.

As already stated above, high triangularity plasmas show larger asymmetries between inner and outer power loads due to part of the energy deposited on the plasma facing components of the upper divertor. At $\nu_{\mathrm{e}}^{*}=0.2-0.5$ about $20 \%$ of the deposited power goes to the inner target plate. In order to check the power balance we have calculated here also the in/out asymmetry for the discharge \#126006, where we do not observe the outer strike zone. As the shape of the plasma has only lower single null, we assume that no power is deposited here to the upper divertor. The value of the energy deposited to the lower outer divertor has been obtained from the following equation:

$$
P_{\text {target }}^{\text {out }}=P_{\text {target }}^{\text {calc }}-P_{\text {target }}^{\text {in }},
$$

where $P_{\text {target }}^{\text {out }}$ is power deposited to the outer target, $P_{\text {target }}^{\text {calc }}$ total power to the divertor obtained from the power balance equation (3) and $P_{\text {target }}^{\text {in }}$ is obtained from the heat flux to the inner target plates measured by the infra-red camera. The data point is marked as circle in Figure 16 and coincides quite well with the other data points for this plasma shape. As we slightly overestimate heat loads to the outer strike point adding there also all the power deposited to the upper divertor, we do expect that this data point would have a little bit higher value of the asymmetry factor. At $\nu_{\mathrm{e}}^{*}>1$ we observe that about $90 \%$ of power coming to the target is deposited through the outer strike zone.

\section{Summary}

Achieving a tolerable heat loads to the plasma facing components are one of the key questions for the safety operation of future fusion devices like ITER or DEMO. As one of the most promising methods to control the power exhaust in poloidally diverted tokamak is the application of RMP, we have performed a review of the experimental results on the heat loads to the lower divertor obtained in DIII-D for different plasma configurations including low and high triangularites and for different electron pedestal collisionalities.

Independent on the plasma triangularity all discharges at electron pedestal collisionality below 0.5 show a slight (about $15 \%$ of the total power) increase of the heat flux to the target plates as compared to the averaged value in non-RMP phase. This seems to be caused by a strong decrease of the floating potential due to very hot electrons from the pedestal area hitting the divertor surface. At very low $\nu_{\mathrm{e}}^{*}$ the hot electrons are able to follow the stochastic field lines up to the target plates. A lower floating potential leads to a higher power deposited by the ions in the sheath and decreases the radiated power in the scrape-off layer. At higher 
collisionalities, high energy electrons dissipate their energy by collisions and do not affect the sheath potential. As a consequence, the total amount of power deposited to the divertor surface is not affected by the RMPs, but we have measured very strong asymmetries between power loads to the inner and outer target.

Also at high collisionalities we observe very clearly striation of the outer strike point with the separation of the lobes strongly exceeding results of the TRIP3D code. The power deposited through the second lobe sometimes exceeds the amount of energy deposited through the main strike line. Both these findings suggest possible enhancement of the external perturbation by its interaction with the plasma. Unfortunately, we still do not fully understand the nature of this coupling, but it is possible that the RMP induces additional currents on the resonant surfaces in the edge. Depending on the relative frequency of the RMP and electron diamagnetic drift frequency they can either suppress or amplify the stochastization of the plasma boundary. At low collisionalities heat and particle flux patterns measured on the inner target plates match, at least qualitatively, with calculated in vacuum magnetic topology of magnetic footprints. Here the asymmetry of power loads between inner and outer target is smaller as compared to higher pedestal collisionalities.

One of the most critical issues for ITER is formed by the transient heat loads to the divertor surface. DIII-D experiments in ITER Similar Shape and with ITER-like electron pedestal collisionalities show that the ELM wetted area monotonically increases with the ELM size expressed as a power deposited to the inner target. There are examples of Type-I ELMs, which show dynamically evolving structures in the first few hundreds of microseconds until the heat loads reach their maximum. Typically, we do observe a few additional substructures separated from each other by $2-3 \mathrm{~cm}$. Typically, after reaching a maximum in the deposited power, the target power does not show any changes of the internal substructures, but decays within $1 \mathrm{~ms}$ to the pre-ELM values. In the initial RMP phase, where still few, smaller ELMs remains, we observe different behavior of the deposition patterns. Small, probably Type-II ELMs deposit all their energy through the main strike line or create one additional, but rather faint substructure. On the other hand, most of the Type-I ELMs show two additional lobes with separation from the main strike line in a fair agreement with the structure of the strike point at this toroidal angle as predicted by vacuum modeling. They also show no evolution characteristic for the ELMs in the non-RMP phase, which could suggest locking of ELMs to the external perturbation.

\section{Bibliography}

[1] H. Zohm, "Edge localized modes (ELMs)," Plasma Physics and Controlled Fusion, vol. 38, 1996, pp. 105-128.

[2] A. Kirk, B. Koch, R. Scannell, H.R. Wilson, G. Counsell, J. Dowling, A. Herrmann, R. Martin, and M.W.M. team, "Evolution of Filament Structures during Edge-Localized Modes in the MAST Tokamak," Physical Review Letters, vol. 96, 2006, pp. 185001-4.

[3] F. Wagner, M. Hirsch, H. Hartfuss, H.P. Laqua, and H. Maassberg, "H-mode and transport barriers in helical systems," Plasma Physics and Controlled Fusion, vol. 48, 2006, pp. A217-A239.

[4] T. Eich, A. Herrmann, J. Neuhauser, R. Dux, J.C. Fuchs, S. Günter, L.D. Horton, A. Kallenbach, P.T. Lang, C.F. Maggi, M. Maraschek, V. Rohde, W. Schneider, and T.A.U. Team, "Type-I ELM substructure on the divertor target plates in ASDEX Upgrade," Plasma Physics and Controlled Fusion, vol. 47, 2005, pp. 815-842.

[5] J. Luxon, "A design retrospective of the DIII-D tokamak," Nuclear Fusion, vol. 42, 2002, pp. 614-633.

[6] K.H. Burrell, T.E. Evans, E.J. Doyle, M.E. Fenstermacher, R.J. Groebner, A.W. Leonard, R.A. Moyer, T.H. Osborne, M.J. Schaffer, P.B. Snyder, P.R. Thomas, W.P. West, J.A. 
Boedo, A.M. Garofalo, P. Gohil, G.L. Jackson, R.J.L. Haye, C.J. Lasnier, H. Reimerdes, T.L. Rhodes, J.T. Scoville, W.M. Solomon, D.M. Thomas, G. Wang, J.G. Watkins, and L. Zeng, "ELM suppression in low edge collisionality H-mode discharges using $\mathrm{n}=3$ magnetic perturbations," Plasma Physics and Controlled Fusion, vol. 47, 2005, pp. B37-B52.

[7] T.E. Evans, R.A. Moyer, K.H. Burrell, M.E. Fenstermacher, I. Joseph, A.W. Leonard, T.H. Osborne, G.D. Porter, M.J. Schaffer, P.B. Snyder, P.R. Thomas, J.G. Watkins, and W.P. West, "Edge stability and transport control with resonant magnetic perturbations in collisionless tokamak plasmas," Nat Phys, vol. 2, 2006, pp. 419-423.

[8] R.A. Moyer, T.E. Evans, T.H. Osborne, P.R. Thomas, M. Becoulet, J. Harris, K. Finken, J.A. Boedo, E.J. Doyle, M.E. Fenstermacher, P. Gohil, R.J. Groebner, M. Groth, G.L. Jackson, R.J. La Haye, C.J. Lasnier, A.W. Leonard, G.R. McKee, H. Reimerdes, T.L. Rhodes, D.L. Rudakov, M.J. Schaffer, P.B. Snyder, M.R. Wade, G. Wang, J.G. Watkins, W.P. West, and L. Zeng, "Edge localized mode control with an edge resonant magnetic perturbation," Physics of Plasmas, vol. 12, 2005, pp. 056119-11.

[9] T. Evans, M. Fenstermacher, R. Moyer, T. Osborne, J. Watkins, P. Gohil, I. Joseph, M. Schaffer, L. Baylor, M. Bécoulet, J. Boedo, K. Burrell, J. deGrassie, K. Finken, T. Jernigan, M. Jakubowski, C. Lasnier, M. Lehnen, A. Leonard, J. Lonnroth, E. Nardon, V. Parail, O. Schmitz, B. Unterberg, and W. West, "RMP ELM suppression in DIII-D plasmas with ITER similar shapes and collisionalities," Nuclear Fusion, vol. 48, 2008, p. 024002.

[10] T.E. Evans, R.A. Moyer, and P. Monat, "Modeling of stochastic magnetic flux loss from the edge of a poloidally diverted tokamak," Physics of Plasmas, vol. 9, 2002, pp. 49574967.

[11] O. Schmitz, T.E. Evans, M.E. Fenstermacher, H. Frerichs, M.W. Jakubowski, M.J. Schaffer, A. Wingen, W.P. West, N.H. Brooks, K.H. Burrell, J.S. deGrassie, Y. Feng, K.H. Finken, P. Gohil, M. Groth, I. Joseph, C.J. Lasnier, M. Lehnen, A.W. Leonard, S. Mordijck, R.A. Moyer, A. Nicolai, T.H. Osborne, D. Reiter, U. Samm, K.H. Spatschek, H. Stoschus, B. Unterberg, E.A. Unterberg, J.G. Watkins, R. Wolf, T. DIII-D, and T. Teams, "Aspects of three dimensional transport for ELM control experiments in ITERsimilar shape plasmas at low collisionality in DIII-D," Plasma Physics and Controlled Fusion, vol. 50, 2008, p. 124029.

[12] M.W. Jakubowski, O. Schmitz, S.S. Abdullaev, S. Brezinsek, K.H. Finken, A. KramerFlecken, M. Lehnen, U. Samm, K.H. Spatschek, B. Unterberg, and R.C.W.T. Team, "Change of the Magnetic-Field Topology by an Ergodic Divertor and the Effect on the Plasma Structure and Transport," Physical Review Letters, vol. 96, (2006), pp. 0350044.

[13] M. Schaffer, J. Menard, M. Aldan, J. Bialek, T. Evans, and R. Moyer, "Study of invessel nonaxisymmetric ELM suppression coil concepts for ITER," Nuclear Fusion, vol. 48, 2008, p. 024004.

[14] M.Z. Tokar and A. Gupta, "Elucidation of the Heat-Flux Limit from Magnetic-Island Heating," Physical Review Letters, vol. 99, 2007, pp. 225001-4.

[15] A. Wingen and K.H. Spatschek, "Ambipolar stochastic particle diffusion and plasma rotation," Physics of Plasmas, vol. 15, 2008, pp. 052305-8.

[16] M. Fenstermacher, T. Evans, T. Osborne, M. Schaffer, M. Aldan, J. Degrassie, P. Gohil, I. Joseph, R. Moyer, P. Snyder, R. Groebner, M. Jakubowski, A. Leonard, and O. Schmitz, "Effect of Island Overlap on Edge Localized Mode Suppression by Resonant Magnetic Perturbations in DIII-D," Physics of Plasmas, vol. 15, 2008. 
[17] T.E. Evans, R.K.W. Roeder, J.A. Carter, B.I. Rapoport, M.E. Fenstermacher, and C.J. Lasnier, "Experimental signatures of homoclinic tangles in poloidally diverted tokamaks," Journal of Physics: Conference Series, vol. 7, 2005, pp. 174-190.

[18] S. Abdullaev, K. Finken, M. Jakubowski, and M. Lehnen, "Mappings of stochastic field lines in poloidal divertor tokamaks," Nuclear Fusion, vol. 46, 2006, pp. S113-S126.

[19] F. Nguyen, P. Chendrih, and A. Grosman, "Interaction of stochastic boundary layer with plasma facing components," Nuclear Fusion, vol. 37, 1997, pp. 743-757.

[20] P. Ghendrih, A. Grosman, and H. Capes, "Theoretical and experimental investigations of stochastic boundaries in tokamaks," Plasma Physics and Controlled Fusion, vol. 38, 1996, pp. 1653-1724.

[21] M. Jakubowski, S. Abdullaev, K. Finken, and T.T. Team, "Modelling of the magnetic field structures and first measurements of heat fluxes for TEXTOR-DED operation," Nuclear Fusion, vol. 44, 2004, pp. S1-S11.

[22] O. Schmitz, T.E. Evans, M.E. Fenstermacher, H. Frerichs, M.W. Jakubowski, M.J. Schaffer, A. Wingen, W.P. West, N.H. Brooks, K.H. Burrell, J.S. deGrassie, Y. Feng, K.H. Finken, P. Gohil, M. Groth, I. Joseph, C.J. Lasnier, M. Lehnen, A.W. Leonard, S. Mordijck, R.A. Moyer, A. Nicolai, T.H. Osborne, D. Reiter, U. Samm, K.H. Spatschek, H. Stoschus, B. Unterberg, E.A. Unterberg, J.G. Watkins, R. Wolf, T. DIII-D, and T. Teams, "Aspects of three dimensional transport for ELM control experiments in ITERsimilar shape plasmas at low collisionality in DIII-D," Plasma Physics and Controlled Fusion, vol. 50, 2008, p. 124029.

[23] T. Evans, J.H. Yu, M.W. Jakubowski, O. Schmitz, J.G. Watkins, and R.A. Moyer, “A conceptual model of the magnetic topology and nonlinear dynamics of ELMs," Journal of Nuclear Materials, vol. to be published.

[24] T.E. Evans, R.K.W. Roeder, J.A. Carter, and B.I. Rapoport, "Homoclinic tangles, bifurcations and edge stochasticity in diverted tokamaks," Contributions to Plasma Physics, vol. 44, 2004, pp. 235-240.

[25] M. Jakubowski, S. Abdullaev, K. Finken, and M. Lehnen, "Heat deposition patterns on the target plates of the dynamic ergodic divertor," Journal of Nuclear Materials, vol. 337-339, Mar. 2005, pp. 176-180.

[26] A. Herrmann, W. Junker, K. Gunther, S. Bosch, M. Kaufmann, J. Neuhauser, G. Pautasso, T. Richter, and R. Schneider, "Energy flux to the ASDEX-Upgrade diverter plates determined by thermography and calorimetry," Plasma Physics and Controlled Fusion, vol. 37, 1995, pp. 17-29.

[27] T. Eich, P. Andrew, A. Herrmann, W. Fundamenski, A. Loarte, R.A. Pitts, and J. contributors, "ELM resolved energy distribution studies in the JET MKII Gas-Box divertor using infra-red thermography," Plasma Physics and Controlled Fusion, vol. 49, 2007, pp. 573-604.

[28] T. Eich, A. Herrmann, and J. Neuhauser, "Nonaxisymmetric Energy Deposition Pattern on ASDEX Upgrade Divertor Target Plates during Type-I Edge-Localized Modes," Physical Review Letters, vol. 91, Listopad. 2003, p. 195003.

[29] H.R. Wilson and S.C. Cowley, "Theory for Explosive Ideal Magnetohydrodynamic Instabilities in Plasmas," Physical Review Letters, vol. 92, Kwiecie. 2004, p. 175006.

[30] T.E. Evans, C. Lasnier, D.N. Hill, A.W. Leonard, M.E. Fenstermacher, T.W. Petrie, and M.J. Schaffer, "Measurements of non-axisymmetric effects in the DIII-D divertor," Journal of Nuclear Materials, vol. 220-222, 1995, pp. 235-239.

[31] C.G. Silva, S.J. Fielding, K.B. Axon, and M.G. Booth, "SOL currents and divertor asymmetries on COMPASS-D," Journal of Nuclear Materials, vol. 266-269, Mar. 1999, pp. 679-684. 
[32] P.J. Harbour, "Current Flow Parallel to the Field in a Scrape-Off Layer," Contributions to Plasma Physics, vol. 28, 1988, pp. 417-419.

[33] T. Eich, A. Kallenbach, R. Pitts, S. Jachmich, J. Fuchs, A. Herrmann, and J. Neuhauser, "Divertor power deposition and target current asymmetries during type-I ELMs in ASDEX Upgrade and JET," Journal of Nuclear Materials, vol. 363-365, Jun. 2007, pp. 989-993.

[34] M. Jakubowski, A. Wingen, S. Abdullaev, K. Finken, M. Lehnen, K. Spatschek, and R. Wolf, "Observation of the heteroclinic tangles in the heat flux pattern of the ergodic divertor at TEXTOR," Journal of Nuclear Materials, vol. 363-365, Jun. 2007, pp. 371376.

[35] A. Wingen, M. Jakubowski, K.H. Spatschek, S.S. Abdullaev, K.H. Finken, M. Lehnen, and T. team, "Traces of stable and unstable manifolds in heat flux patterns," Physics of Plasmas, vol. 14, Apr. 2007, pp. 042502-7.

[36] M.E. Fenstermacher, A.W. Leonard, P.B. Snyder, J.A. Boedo, N.H. Brooks, R.J. Colchin, D.S. Gray, R.J. Groebner, M. Groth, E. Hollmann, C.J. Lasnier, T.H. Osborne, T.W. Petrie, D.L. Rudakov, H. Takahashi, J.G. Watkins, L. Zeng, and T.D. Team, "ELM particle and energy transport in the SOL and divertor of DIII-D," Plasma Physics and Controlled Fusion, vol. 45, 2003, pp. 1597-1626.

[37] I. Joseph, T. Evans, A. Runov, M. Fenstermacher, M. Groth, S. Kasilov, C. Lasnier, R. Moyer, G. Porter, M. Schaffer, R. Schneider, and J. Watkins, "Calculation of stochastic thermal transport due to resonant magnetic perturbations in DIII-D," Nuclear Fusion, vol. 48, 2008, p. 045009.

[38] J.G. Watkins, T.E. Evans, C. Lasnier, R.A. Moyer, and D.L. Rudakov, "Target plate conditions during stochastic boundary operation on DIII-D," Journal of Nuclear Materials, vol. 363 - 365, 2007, pp. 708 - 712.

[39] M.W. Jakubowski, M. Lehnen, K.H. Finken, O. Schmitz, S.S. Abdullaev, B. Unterberg, and R. Wolf, "Influence of the Dynamic Ergodic Divertor on the heat deposition pattern in TEXTOR at different collisionalities," Plasma Physics and Controlled Fusion, vol. 49, 2007, pp. S109-S121.

[40] T. Evans, I. Joseph, R. Moyer, M. Fenstermacher, C. Lasnier, and L. Yan, "Experimental and numerical studies of separatrix splitting and magnetic footprints in DIII-D," Journal of Nuclear Materials, vol. 363-365, Jun. 2007, pp. 570-574.

[41] O. Schmitz, M.W. Jakubowski, H. Frerichs, D. Harting,, M. Lehnen, B. Unterberg, S.S. Abdullaev, S. Brezinsek, I. Classen, T.E. Evans, Y. Feng, K.H. Finken, M. Kantor, D. Reiter, U. Samm, B. Schweer, G. Sergienko, G.W. Spakman, M. Tokar, E. Uzgel, and R. Wolf, "Identification and analysis of transport domains in the stochastic boundary of TEXTOR-DED for different mode spectra," Nuclear Fusion, vol. 48, 2008, p. 024009.

[42] W. Fundamenski, R.A. Pitts, and J.E. contributors, "A model of ELM filament energy evolution due to parallel losses," Plasma Physics and Controlled Fusion, vol. 48, 2006, pp. 109-156.

[43] I.H. Hutchinson, B. LaBombard, J.A. Goetz, B. Lipschultz, G.M. McCracken, J.A. Snipes, and J.L. Terry, "The effects of field reversal on the Alcator C-Mod divertor," Plasma Physics and Controlled Fusion, vol. 37, 1995, pp. 1389-1406. 
(a)

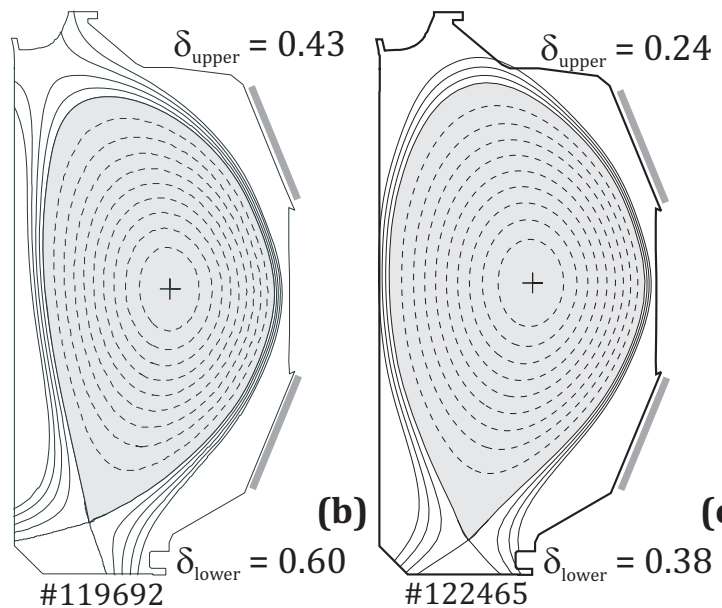

(c)

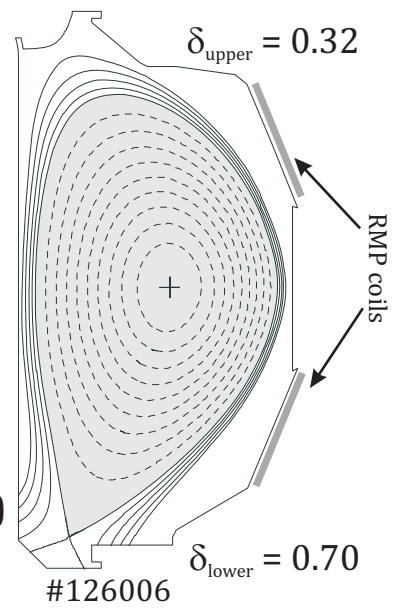

Figure 1. Examples of plasma shapes used to carry out H-mode RMP experiments: a-b) prior to February 2006, c) after February 2006
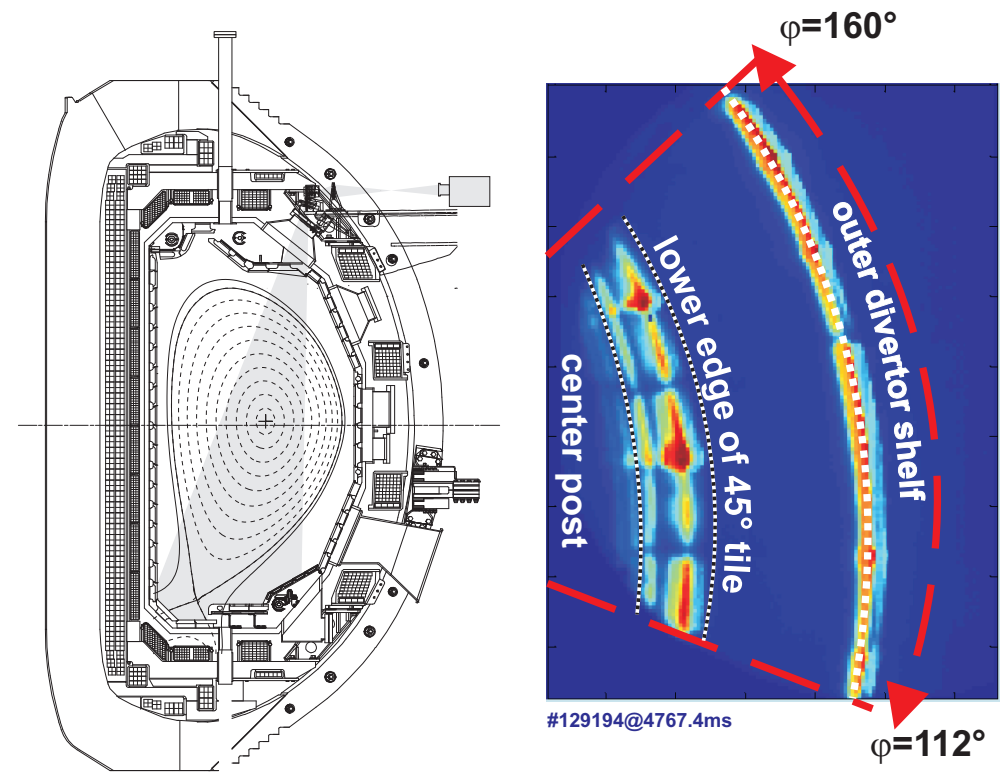

Figure 2. a) Sketch presenting infra-red setup allowing observation of lower divertor overlaid with equilibrium of the DIII-D discharge \#129197. b) Snapshot of the lower divertor during an ELM event taken by the infra-red camera SBF-125. 


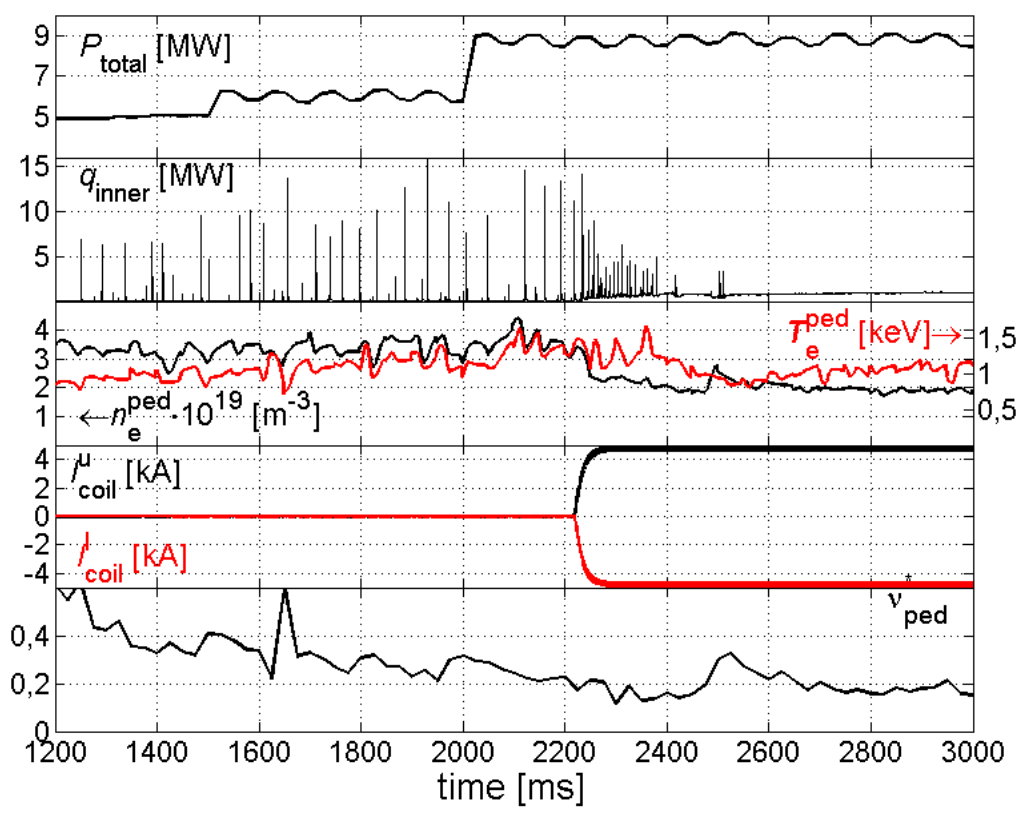

Figure 3. Overview of plasma parameters for the discharge \#129197. From top of the graph: total heating power, power to the inner divertor, electron pedestal temperature and density, Icoil current and electron pedestal collisionality. 


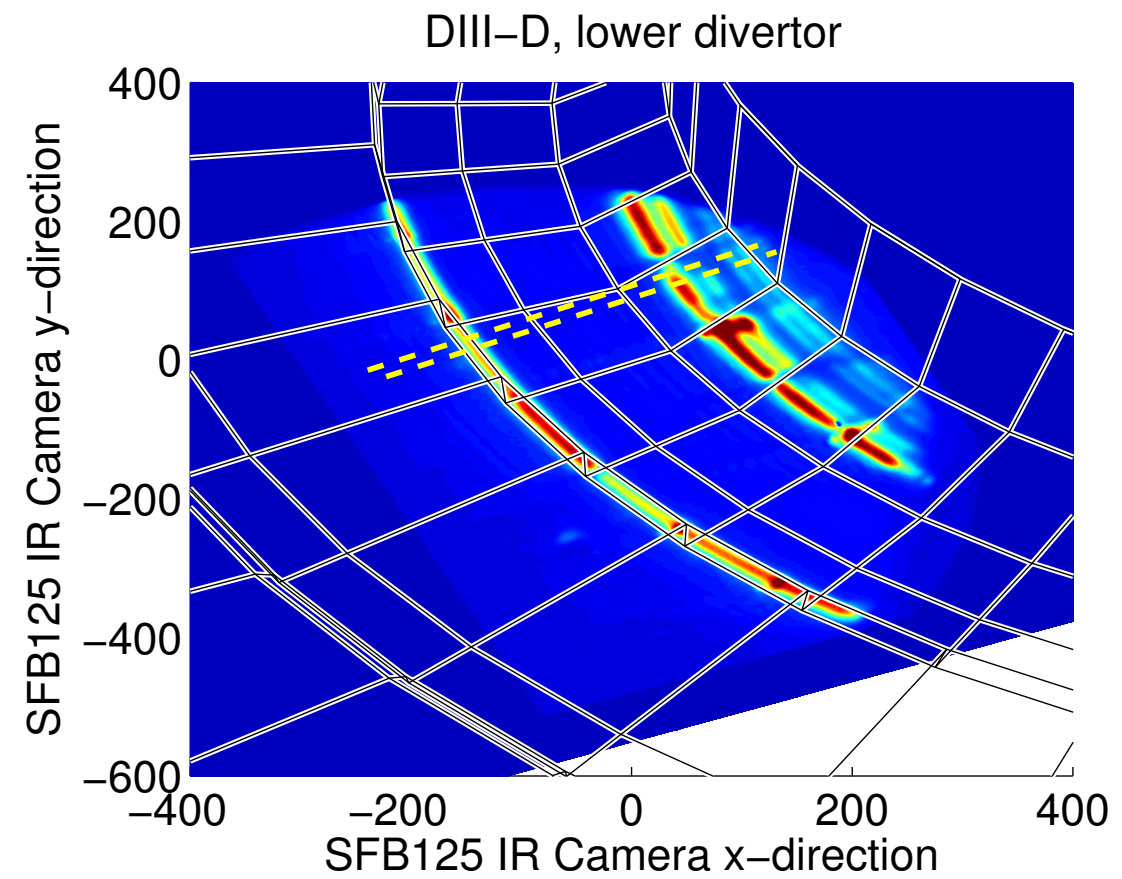

Figure 4. Infra-red image of the lower divertor taken during a Type-I ELM event during the discharge \#129194. Yellow dashed lines indicate area, where heat flux density is evaluated. Color scale has been adapted in order to visualise. 
a)

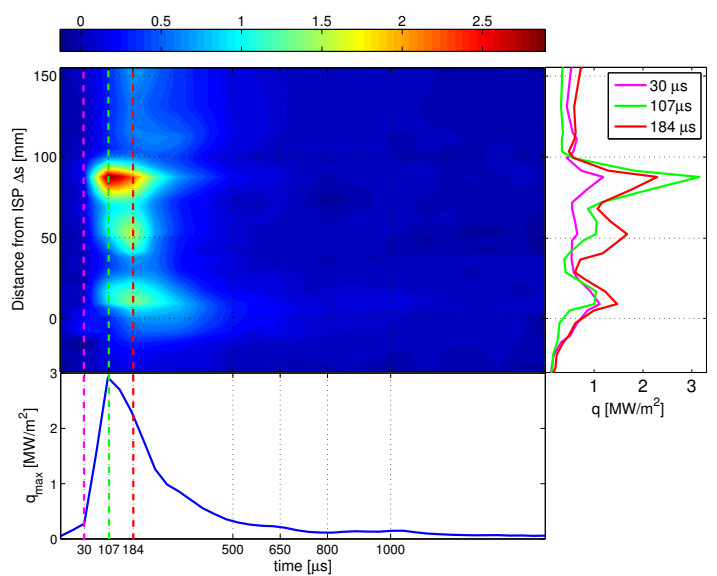

b)

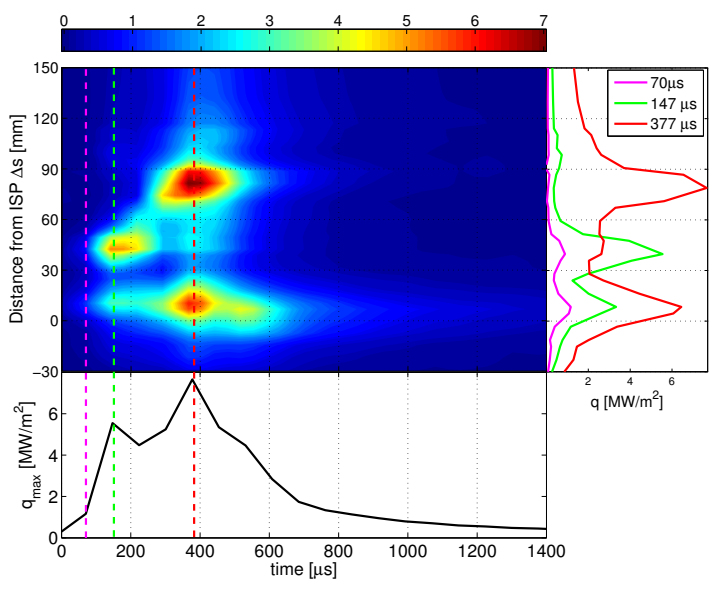

Figure 5. Evolution of the heat flux density at the inner strike point during two Type-I ELMs without RMP during the discharge \#129197: (top) contour plot of heat flux density evolution at the inner strike point in false color representation (units in $\mathrm{MW} / \mathrm{m}^{2}$ ); (bottom) peak heat flux density vs. time for the same event. On the right hand side profiles across the heat flux density are show with times indicated by the dashed lines. 


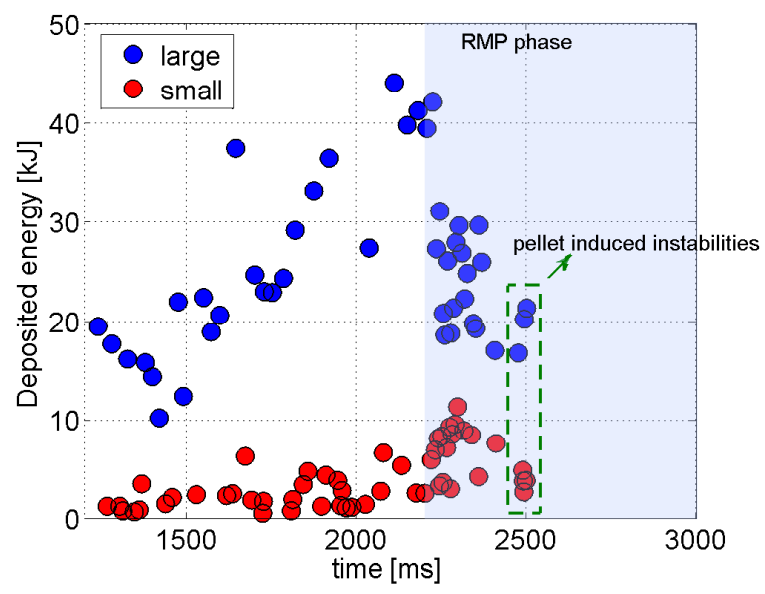

Figure 6. Energy deposited to the inner target due to ELMs during the discharge \#129197. Each data point corresponds to one ELM event.

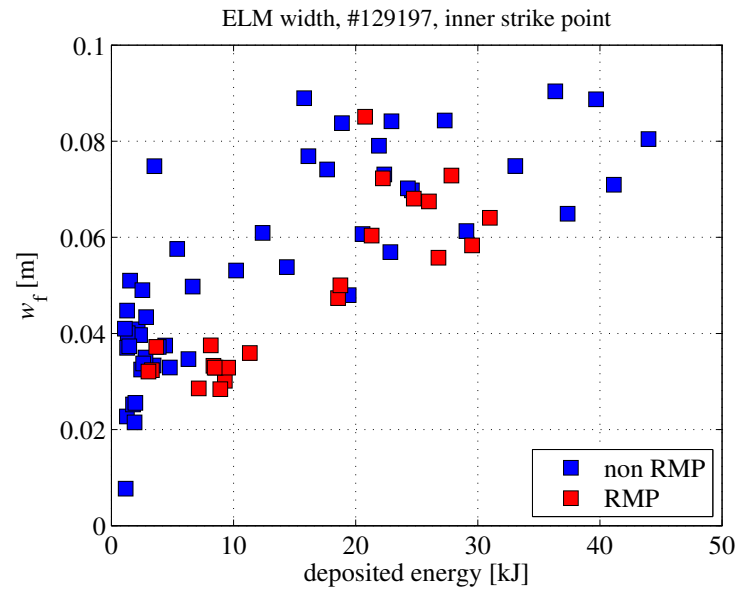

Figure 7. Wetted area of ELMs versus their size in terms of deposited energy to the inner target. 

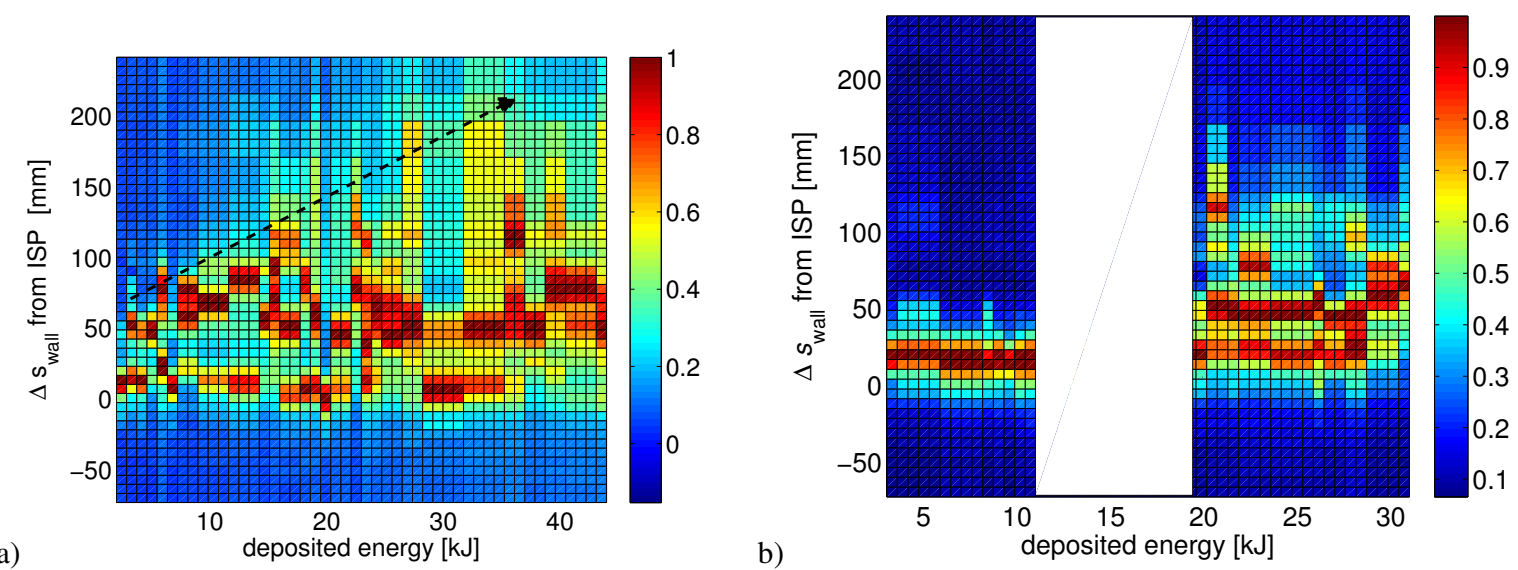

Figure 8. Profiles of ELM heat flux normalized to the peak heat flux value during non-RMP phase of the discharge \#129197 a) and during initial RMP phase b) of the same discharge. Two groups of ELMs can be distinguished by the deposited energy (each vertical line corresponds to one ELM event). No ELMs at all were detected in the energy range inside of the white box.

a)

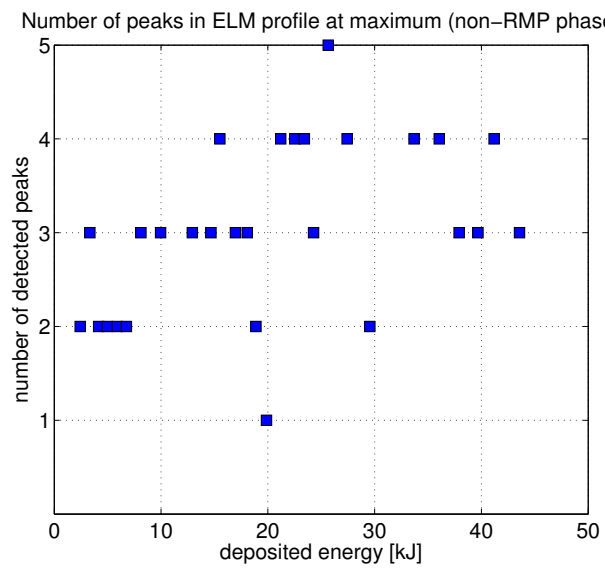
same discharge as in Figure 8.

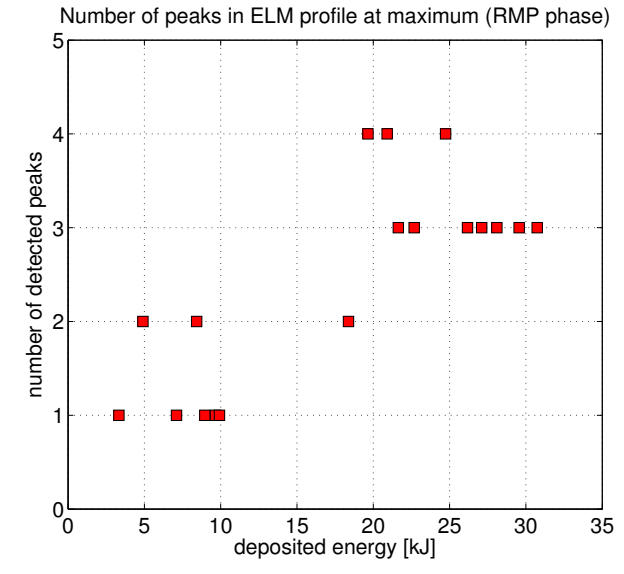

b) 


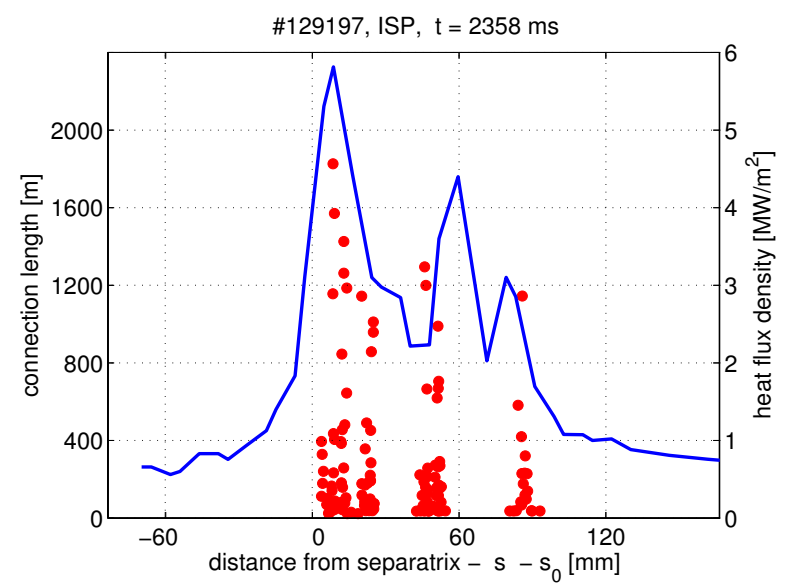

Figure 10. Comparison of heat flux deposition pattern (blue curve, right ordinate) with predicted by TRIP3D connection length distribution (red dots, left ordinate).

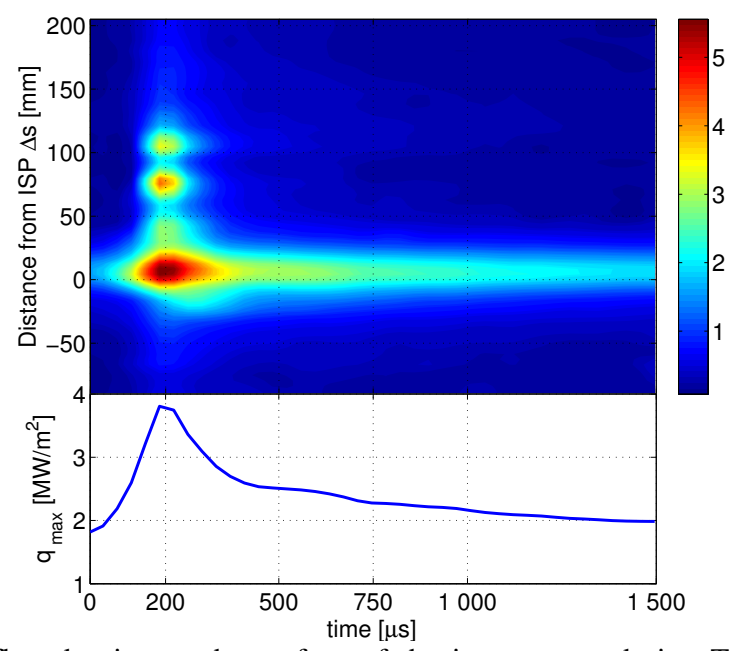

Figure 11. Evolution of heat flux density on the surface of the inner target during Type-I ELM in RMP phase of the discharge \#129197: (top) contour plot of heat flux density evolution at the inner strike point in false color representation (units in $\mathrm{MW} / \mathrm{m}^{2}$ ); (bottom) peak heat flux density vs. time for the same event. 


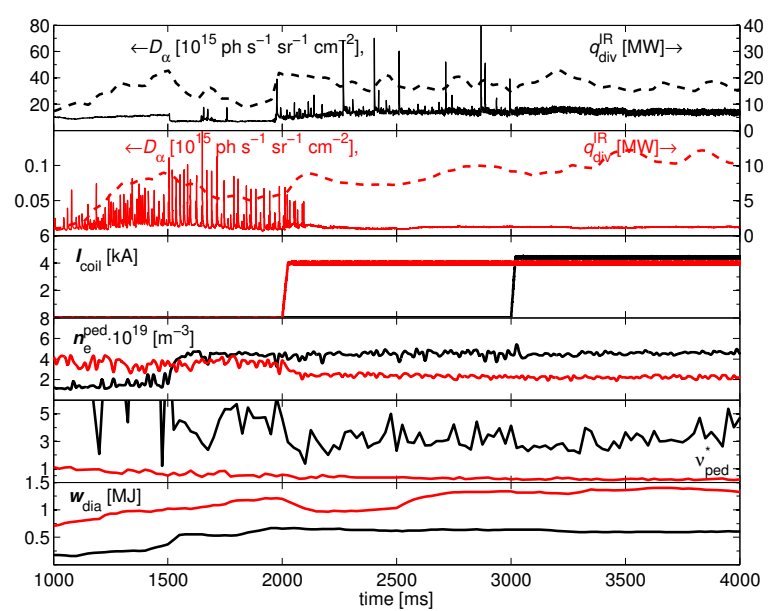

a)

Figure 12. a) Plasma parameters for the discharges a) \#119692 (black curves), 126006 (red curves). From top of the graph: divertor particle (solid) and heat flux (dashed) on top two graphs, I-coil current, pedestal electron density, pedestal collisionality, plasma stored energy. b) Floating potential measured by the Langmuir probe near the outer strike point for the same discharges.

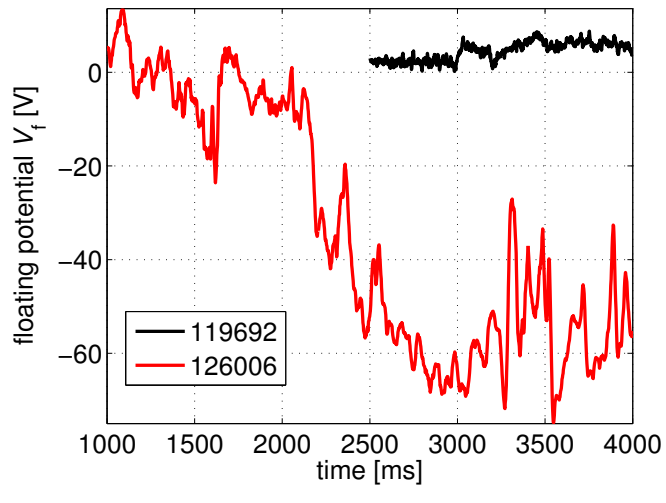

) 

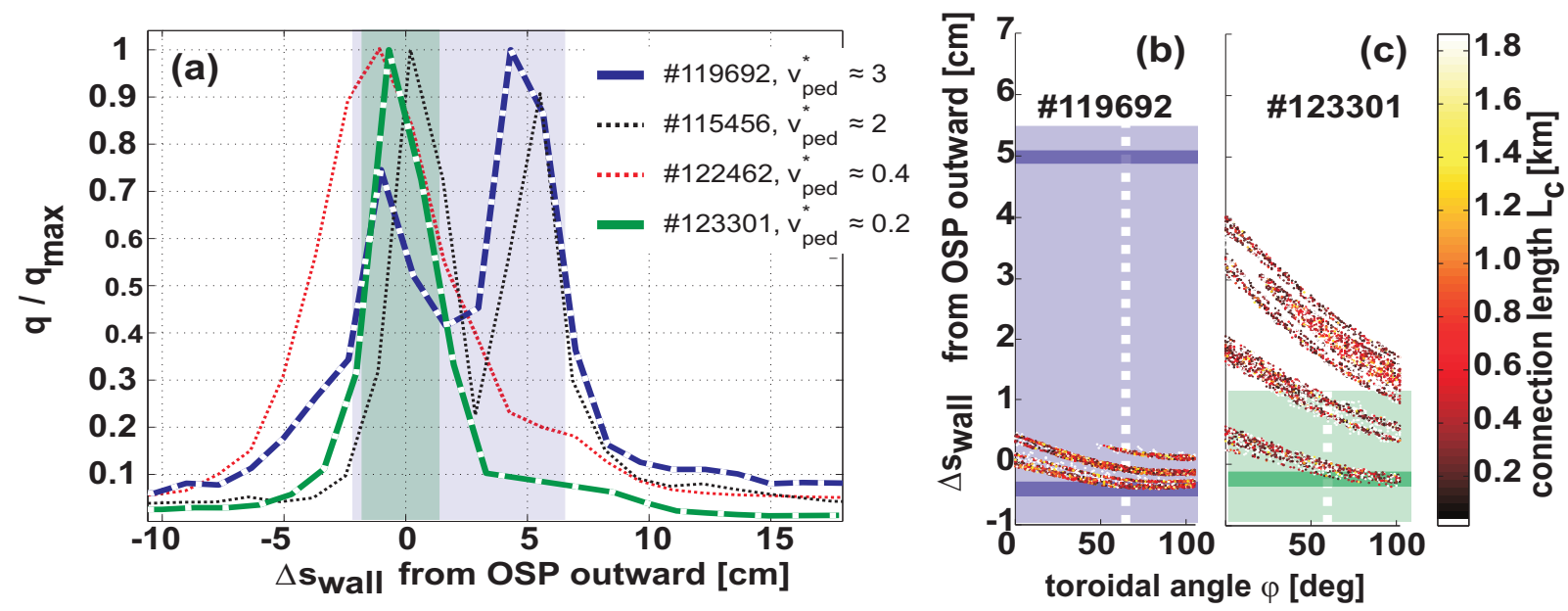

Figure 14. a) Profiles of the heat flux density measured by the infrared camera at the OSP. Profiles are taken during discharges with different collisionalities (see legend). Shaded areas indicate half width of the profiles (\#119692 - purple, \#123301 - green).b-c) Calculated with TRIP3D structure of the magnetic footprints on the divertor surface for \#119692 (b) and \#123301 (c). Color scale denotes connection length $\left(L_{\mathrm{c}}\right)$ of the field lines intersecting the target plates. Green and purple shaded areas indicate width of the experimentally obtained heat flux profiles from (a)
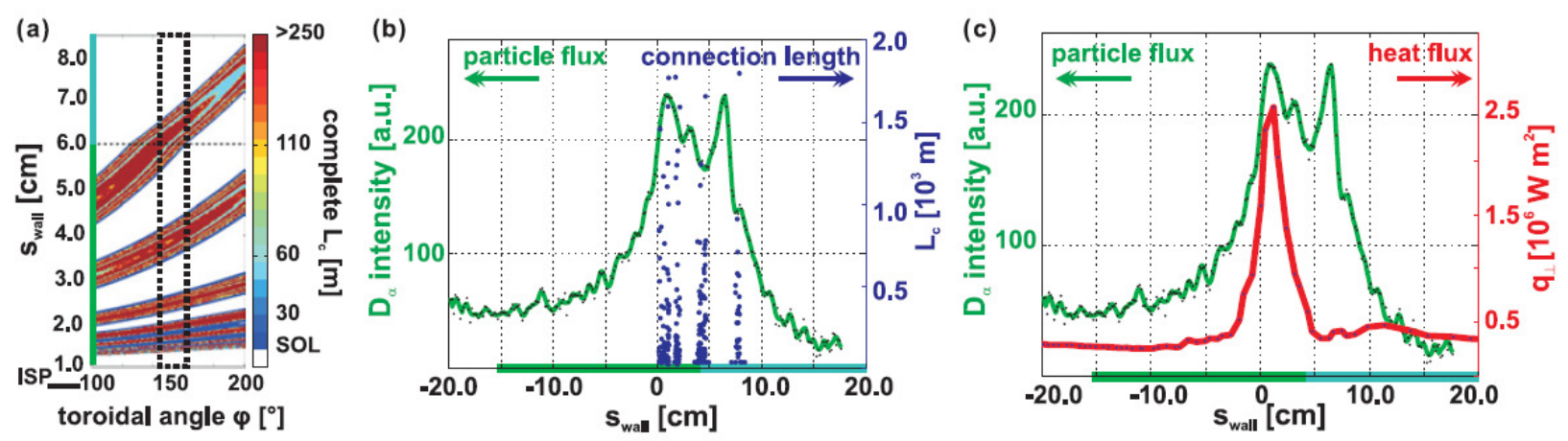

Figure 15. Identification of the perturbed separatrix striations on the inner divertor footprint pattern (reproduced from [22]) A cut of the magnetic footprint $L_{\mathrm{c}}\left(\varphi, s_{\text {wall }}\right)$. b) A direct comparison of the particle flux profile (measured as $D \alpha$ intensity) with $L_{\mathrm{c}}\left(s_{\text {wall }}\right)$. (c) Depicts a direct comparison of the measured particle (green curve) and heat flux (red curve) profiles at the same toroidal angle. 


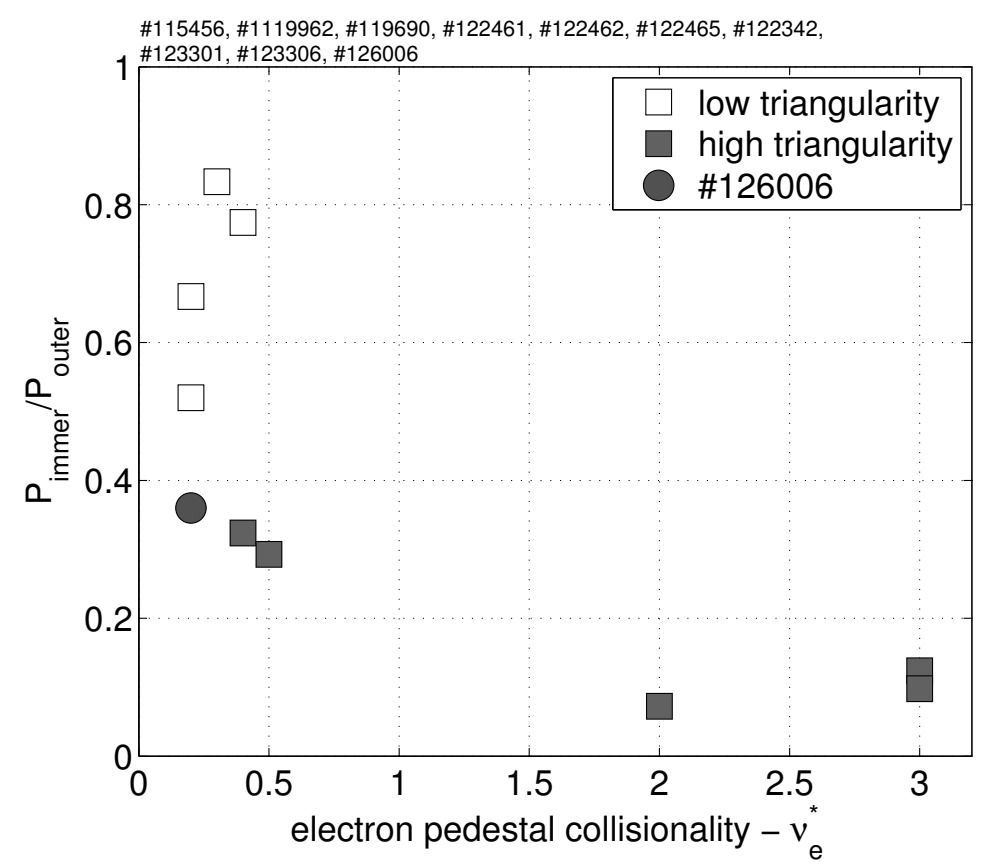

Figure 16. Asymmetries between power loads to the inner and outer strike line in the ELM suppressed phase of the discharge at different collisionalities and triangularities. 